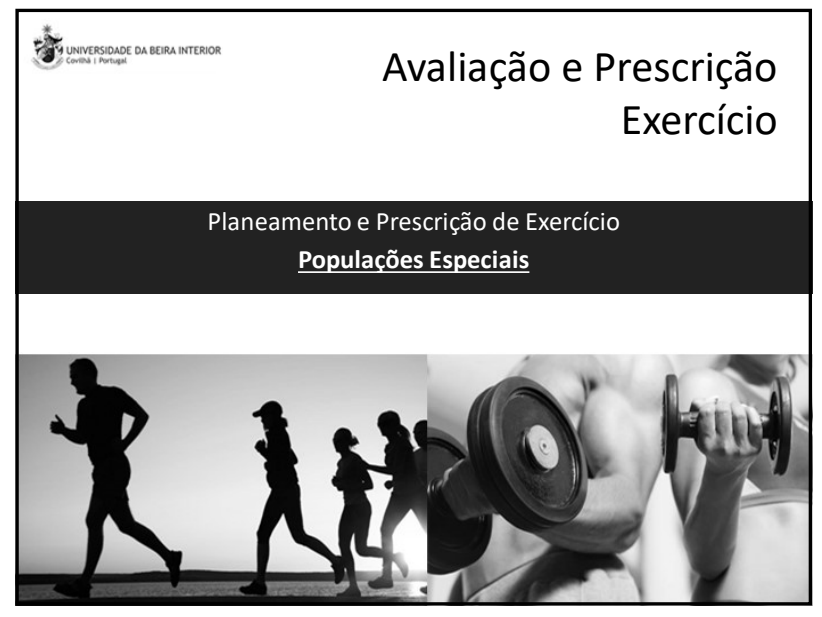

\begin{tabular}{|l|}
\hline Populações Especiais \\
Conteúdos \\
3. Planeamento e Prescrição do Exercício em Populações Especiais \\
3.1. Grávidas \\
3.2. Adultos; idosos \\
3.3. Pré-adolescentes \\
3.4. Problemas nutricionais e metabólicos \\
\hline
\end{tabular}

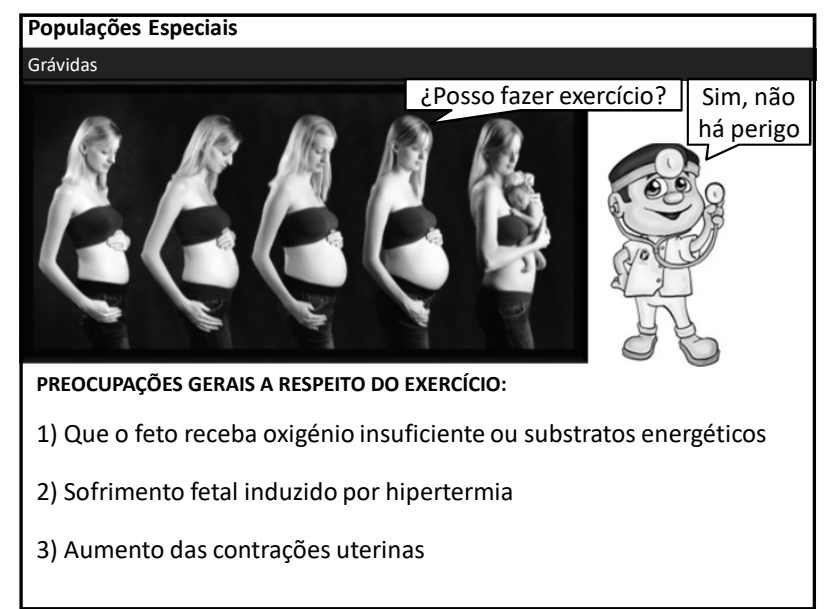




\section{Populações Especiais}

Grávidas: Benefícios

Melhoria da forma física cardiovascular e muscular

Recuperação mais rápida do parto

Recuperação mais rápida do peso, níveis de força e de flexibilidade

Ventre de menores dimensões em puerpério

Redução das dores de costas durante a gravidez

Mais reservas de energia

Menos intervenções obstétricas

Fase mais curta de contrações e menos dor

Menor aumento do peso

Melhoria do bem-estar psicológico da mãe

Aumento da posibilidade de adotar hábitos saudáveis permanentes

Prevenção da diabetes gestacional
Populações Especiais

Grávidas: Alterações Durante a Gravidez

Resposta cardiovascular

- Alteração da frequência cardíaca $\rightarrow$ Escala esforço percebido (12-16)

Síndrome de hipotensão supina $\rightarrow$ Elimina exercicios em supinação (ex. crunch, press peito,

exercícios em decúbito sobre fitball e alongamentos)

Evitar manobra de Valsalva $\rightarrow$ Excessiva pressão sobre o abdómen e bacia $\rightarrow$ Expirar na fase concêntrica do movimento

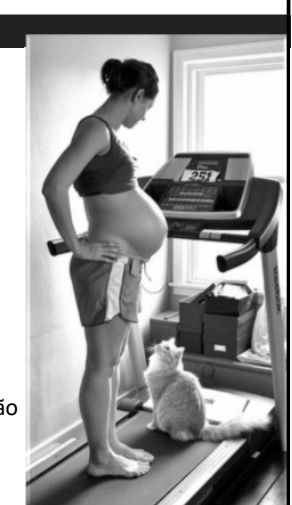

Populações Especiais

\section{Grávidas: Alterações Durante a Gravidez}

Resposta respiratória

- Aumento da ventilação/minuto quase $50 \% \rightarrow$ menos $\mathrm{O}_{2}$ para musculatura $\rightarrow$ Evitar chegar ao esgotamento

\section{Resposta mecânica}

- Alteração do centro de gravidade $\rightarrow$ menor equilíbrio $\rightarrow$ evitar exercício com risco de queda (ex. curl bíceps na máquina vs. halteres de pé; press sentado)

- Evitar atividades com risco de traumatismo abdominal (ex. tenis, esquí...)

- Fortalecer músculos abdominal e pubococcígeo (exercício de Kegel)

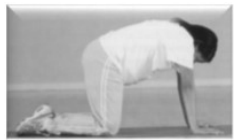




Populações Especiais
Grávidas: Alteraçôes Durante a Gravidez
Resposta metabólica
Maior necessidade de $\mathrm{O}_{2}$ e maior utilização de $\mathrm{HCO} \rightarrow+300 \mathrm{kcal} /$ día
Resposta termorreguladora
adequadar metabolismo basal e maior produção de calor $\rightarrow$ hidratação

Populações Especiais
Grávidas: Interrompemos o exercíio se...
Tangramento vaginal
Taquicardia
Elevação do pulso ou tensão durante/após exercício

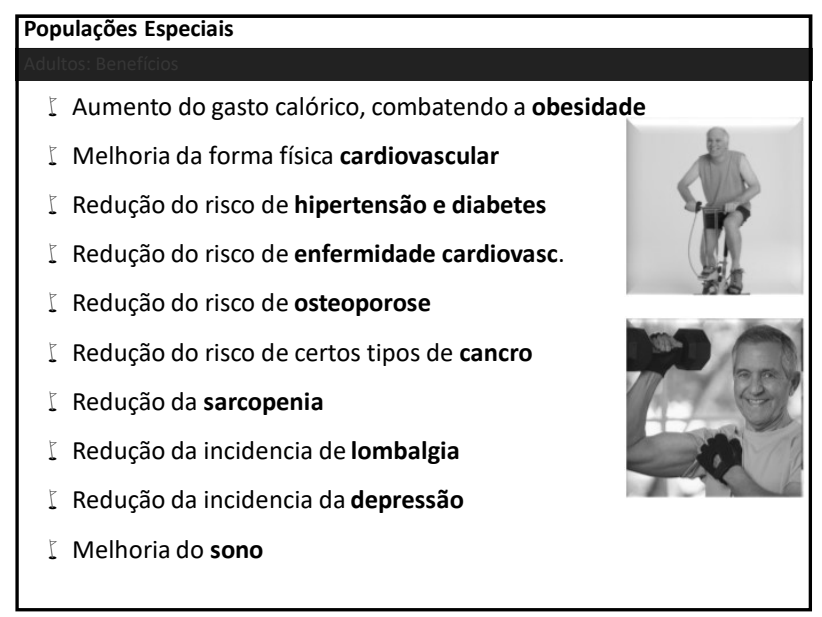




\begin{tabular}{|c|c|c|}
\hline \multicolumn{3}{|c|}{ Populações Especiais } \\
\hline \multicolumn{2}{|r|}{ Treino aeróbio } & \\
\hline Frequência & 2-5 dias / semana & \\
\hline Volume & 20-60 minutos / sessão & \\
\hline Intensidade & $\begin{array}{l}75 \% \text { da } \mathrm{FC}_{\text {máx. }}(\mathrm{PSE}=12-14) \\
\text { Teste da fala }\end{array}$ & \\
\hline \multicolumn{2}{|r|}{ Treino da força } & \\
\hline Frequência & 2-3 dias / semana (não consecutivos) & Exercício aeróbio \\
\hline Volume & 4-16 reps / serie & Exercício força \\
\hline Intensidade & Graduais. Começar com autocarga & \\
\hline № exercícios & Ao menos 1/grupo muscular (Total: 8-10) & $\begin{array}{l}\text { Alongamentos } \\
\text { estáticos }\end{array}$ \\
\hline $\begin{array}{l}\text { Velocidade } \\
\text { execução }\end{array}$ & Controlada & \\
\hline
\end{tabular}

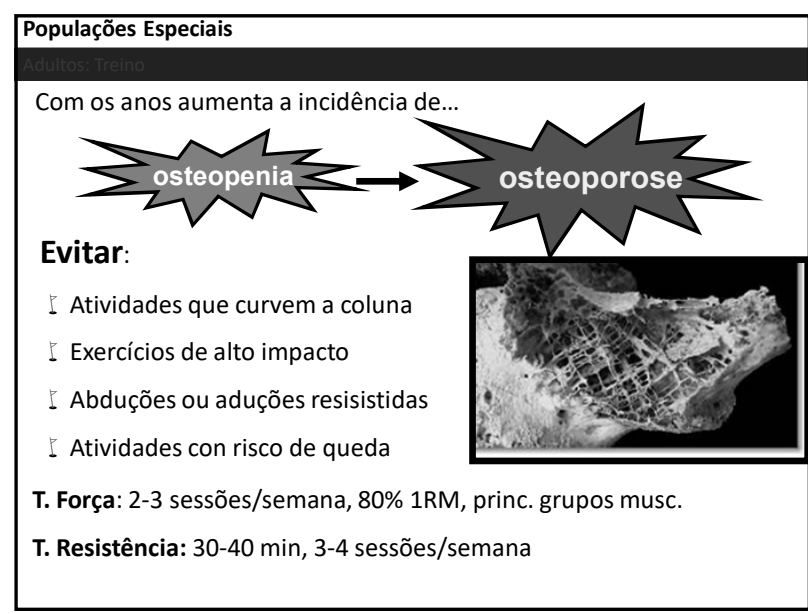

\section{Populações Especiais

Pré-Adolescentes $(6-11 \uparrow, 6-13 \uparrow)$ \\ ACEITAÇÃO DO EXERCÍCIO}

Organizações que afirmam que apoiam a participação das crianças na atividade física (incluindo treino da força):

'Y'American Academy of Pediatrics (1996)

'Y'American College of Sports Medicine (1993)

' $Y$ ' American Orthopedic Society for Sports Medicine (1988)

' $Y$ ' National Strength and Conditioning Association (1996)

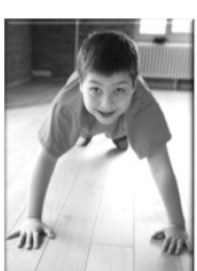




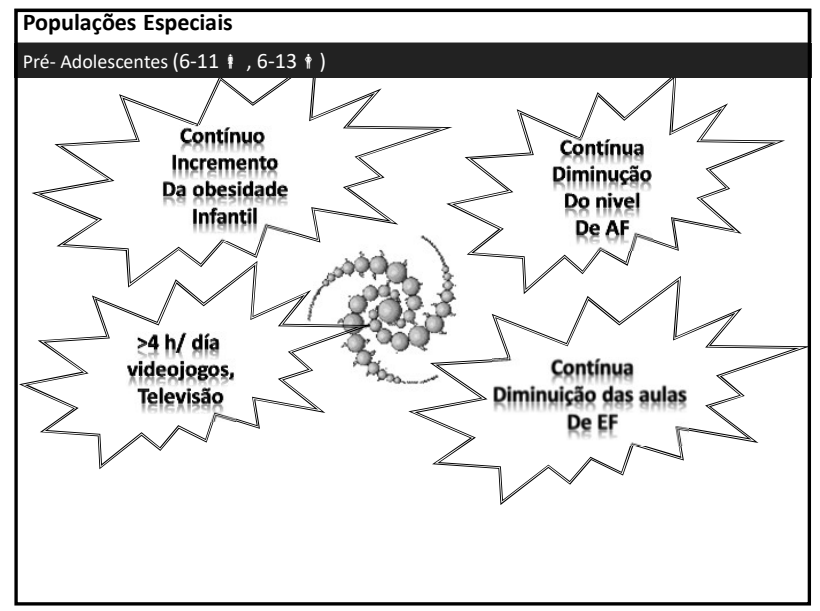

Populações Especiais

\section{Pré-Adolescentes $(6-11 \downarrow, 6-13 \uparrow)$}

- As orientações para adultos não devem ser utilizadas em crianças:

"uma criança não é um adulto pequeno"

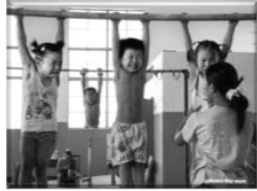

- A atividade natural sofre modificações bruscas de intensidade.

- Nas crianças predomina o pensamento concreto, no lugar do abstrato.

(1)

$\longrightarrow$ Grandes treinos aeróbios podem diminuir a motivação e aderência ao program

\section{Populações Especiais}

Pré-Adolescentes $(6-11\}, 6-13 \uparrow)$

MODELO DE ATIVIDADE FÍSICA

Padrão mínimo de saúde:

Frequência 3 sessões/semana

Intensidade moderada

\begin{tabular}{l|l} 
Duração & A necessária para gastar 3-4 kcal/Kg/dia
\end{tabular}

Padrão funcional ótimo:

Frequência 3 sessões/semana ou +

Intensidade Moderada a vigorosa

Duração $\quad$ A necessária para gastar 6-8 kcal/Kg/dia 


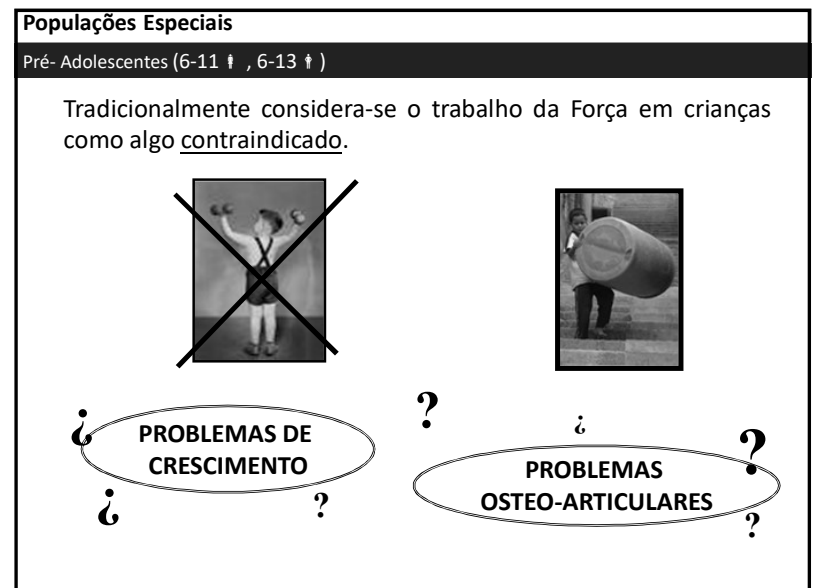

Populações Especiais

Pré-Adolescentes (6-11 , 6-13 \$): Benefícios do treino Força

- Ganho de F muscular, potência e força resistência

- Redução das lesões durante atividades desportivas e lazer

- Melhoria do rendimento em atividades desportivas e lazer

- Aumenta a densidade óssea

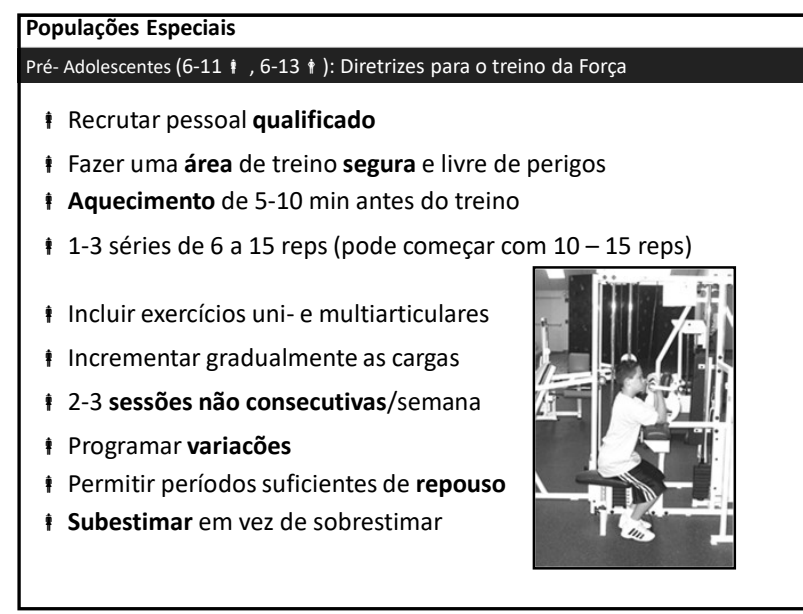




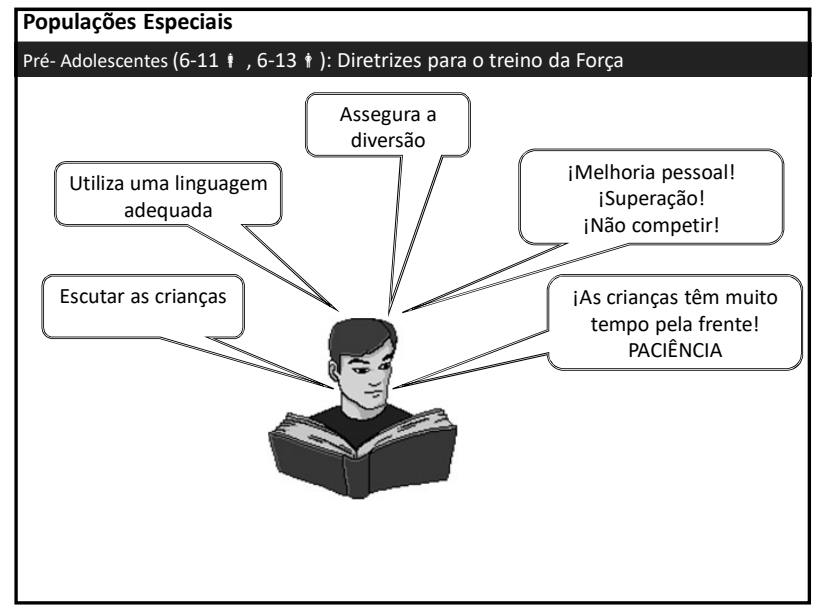

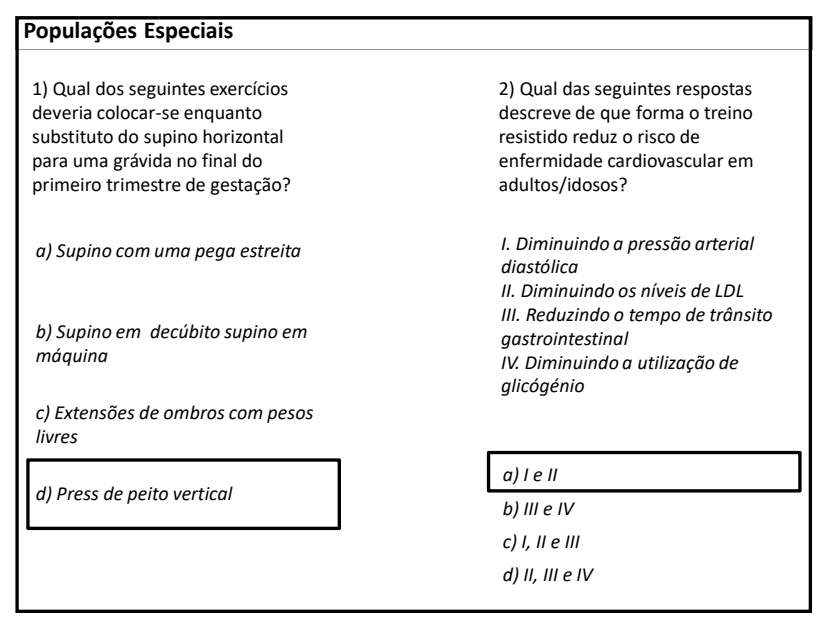

\begin{tabular}{|c|c|}
\hline Populações Especiais & \\
\hline $\begin{array}{l}\text { 3) Como deveríamos modificar a } \\
\text { intensidade de uma sessão de } \\
\text { exercício aeróbio para um adulto } \\
\text { saudável que refere um valor de } \\
\text { PSE } 10 \text { na escala original, sendo que } \\
\text { apresenta uma frequência cardíaca } \\
\text { de } 80 \% \text { da frequência cardíaca } \\
\text { máxima estimada para a idade? }\end{array}$ & $\begin{array}{l}\text { 4) Qual das seguintes respostas é } \\
\text { uma recomendação para uma } \\
\text { criança que quer iniciar um } \\
\text { programa de treino resistido? }\end{array}$ \\
\hline $\begin{array}{l}\text { a) Mantendo a mesma intensidade } \\
\text { de exercício }\end{array}$ & $\begin{array}{l}\text { b) Evitar exercícios multiarticulares } \\
\text { porque impoem uma tensão } \\
\text { excesiva nas articulacões }\end{array}$ \\
\hline \multirow{2}{*}{$\begin{array}{l}\text { b) Reduzindo a intensidade de } \\
\text { exercicio } \\
\text { c) Aumentando a intensidade de } \\
\text { exercício }\end{array}$} & $\begin{array}{l}\text { c) Programar pelo menos um dia de } \\
\text { descanso entre sessões de exercício }\end{array}$ \\
\hline & $\begin{array}{l}\text { d) Dizer a ela e seus país de que } \\
\text { precisa de esperar uns anos mais } \\
\text { para começar um programa de } \\
\text { treino de força }\end{array}$ \\
\hline
\end{tabular}



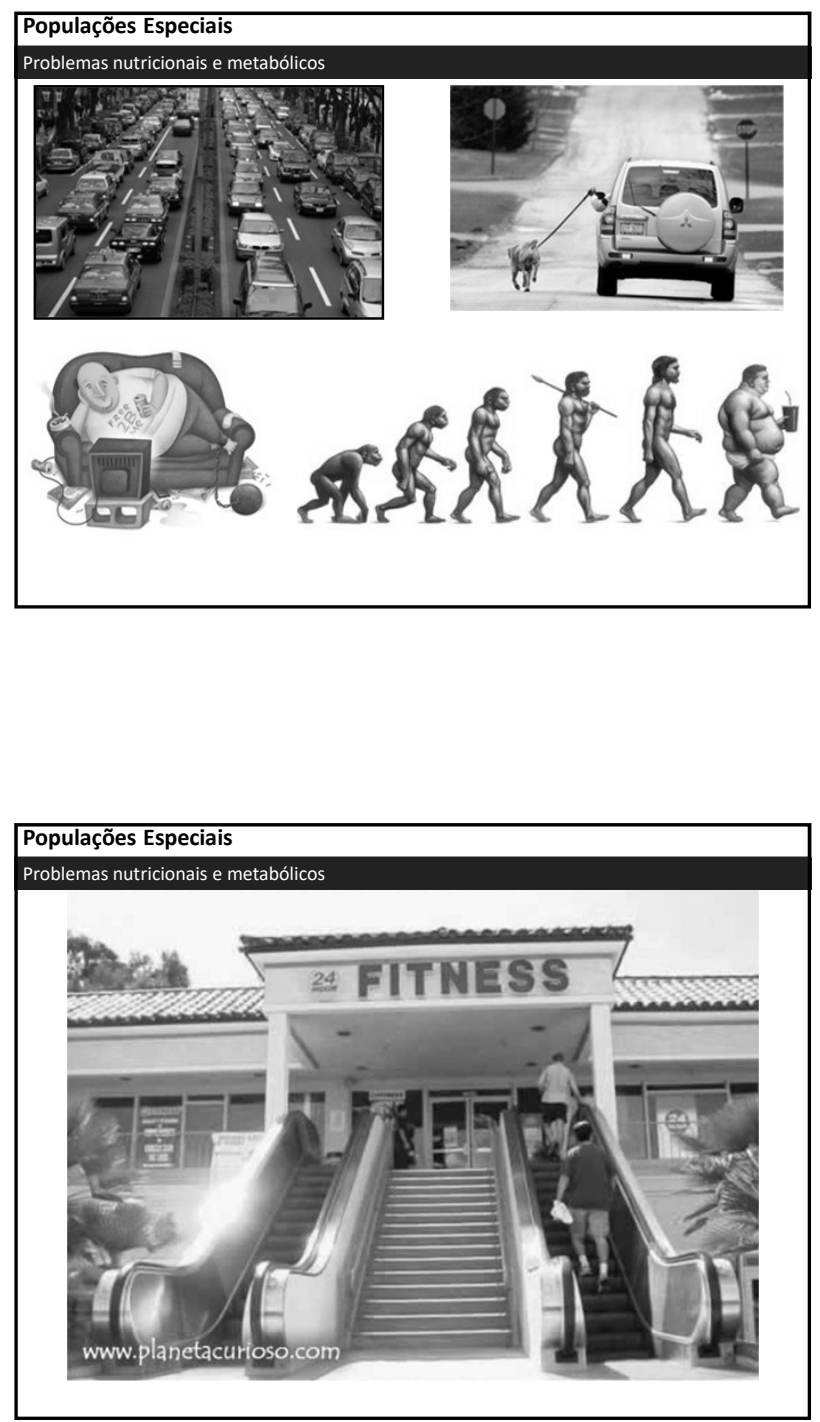

\section{Populações Especiais}

Ao longo do século passado, o nível de atividade física nos países desenvolvidos desceu consideravelmente. Neste período de tempo, o nível de incidência de transtornos alimentares, sobrepeso, obesidade, hiperlipidemia e diabetes aumentou de forma notável.

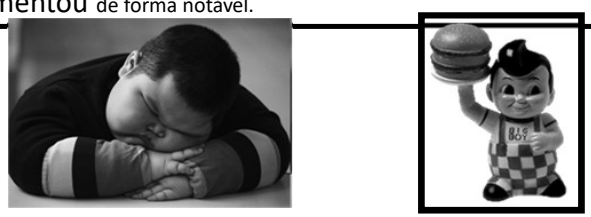

O monitor deve perceber estas desordens e pedir conselho ao médico caso necessário

O diagnóstico destas desordens não é tarefa do treinador pessoal

O controlo médico e dietético debe ser levado a cabo por um profissiona competente. 


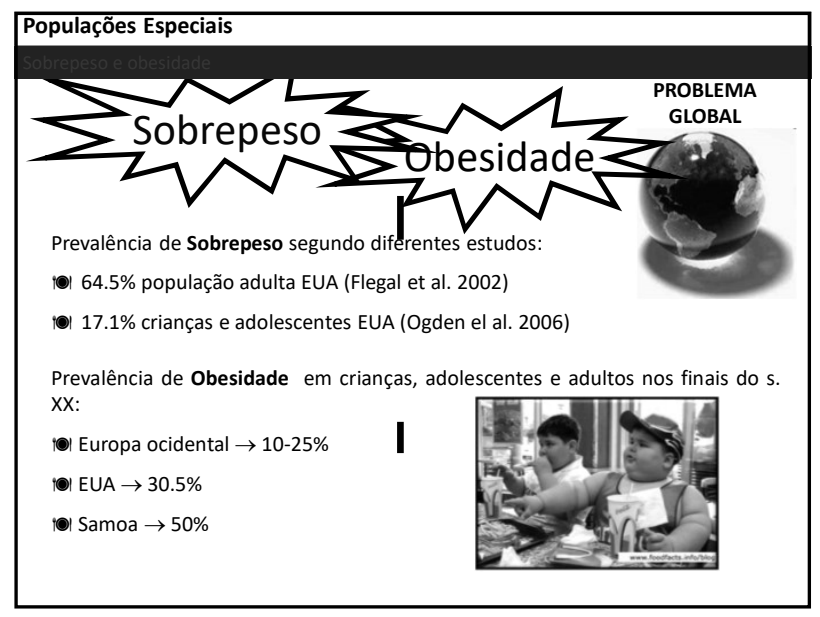

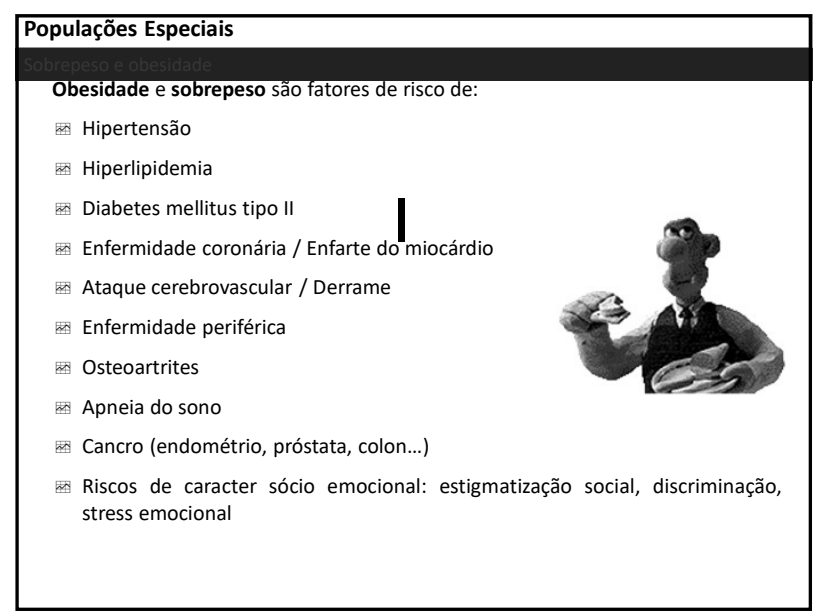

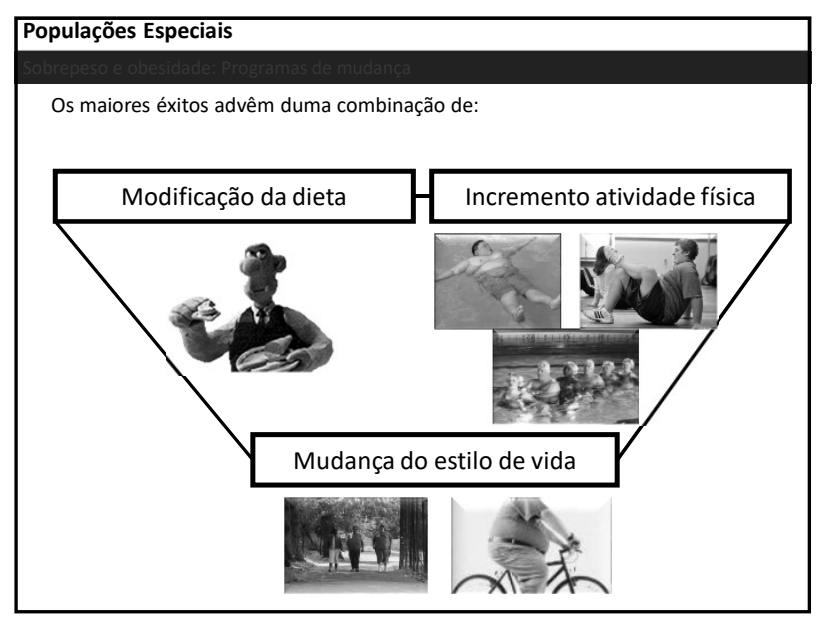




\begin{tabular}{|l|}
\hline \multicolumn{2}{|c|}{ Populações Especiais } \\
$\checkmark$ Deve fazê-la um nutricação da dieta \\
$\checkmark$ A derivação ao nutricionista é recomendável em pacientes \\
obesos com colesterol elevado, e necessária em pacientes com \\
diabetes. \\
$\checkmark$ Recomenda-se gerar um deficit de 500 a 1000 cal/dia. \\
$\checkmark$ As mulheres não deveriam seguir dietas inferiores a 1000- \\
1200 cal/dia e os homens $1200-1600$ cal/dia (<30\% gordura). \\
$\checkmark$ Um objetivo realista e alcançável para a maioria dos clientes \\
pode ser uma perda de 10\% do peso inicial em $\mathbf{6}$ meses. Após os \\
6 meses devemos estabelecer novos objetivos. \\
\end{tabular}

\begin{tabular}{|l|}
\hline \multicolumn{1}{|c|}{ Populações Especiais } \\
\hline \multicolumn{1}{|c|}{ Incremento da atividade física } \\
- Intolerância ao calor, restrição de movimentos, stress \\
derivado do seu próprio peso, problemas posturais, dôr \\
lombar, alterações do equilíbrio, apneia e dispneia. \\
- O objetivo é mantêr ou melhorar a massa livre de gordura. \\
- Atenção a problemas lombares e articulares: modificar e \\
adaptar exercícios. \\
- Começar só com exercícios de autocargas. \\
- Enfatizar a força da componente superior (o cliente obeso \\
tem usualmente suficiente força no "trem" inferior). \\
- Enfatizar em exercícios que impliquem grandes grupos \\
musculares, para incrementar o gasto calórico (de 300-500 \\
kcal/dia). \\
- Táticas motivacionais!
\end{tabular}

\begin{tabular}{|c|c|}
\hline \multicolumn{2}{|c|}{ Populações Especiais } \\
\hline & Incremento da atividade física \\
\hline \multicolumn{2}{|c|}{ Prescrição do exercício para clientes obesos: } \\
\hline \multicolumn{2}{|l|}{ Treino aeróbio: } \\
\hline Frequência & 5 dias/semana (ou diário). \\
\hline Volume & 1-2 sessões $20-30 \mathrm{~min}$. Objetivo final: $40-60 \mathrm{~min} / \mathrm{dia}$ \\
\hline Tipo de exercício & Cuidado lesões ortopédicas. Treino intermitente \\
\hline Intensidade & $40-70 \% \mathrm{VO}_{2} \max$ (incrementar antes o volume) \\
\hline \multicolumn{2}{|c|}{ Treino da força (Incremento da TMB) } \\
\hline Frequência & 2-3 dias/semana (não consecutivos) \\
\hline Volume & 1-3 séries, $10-15$ rep/série (resistência muscular) \\
\hline No exercícios & Até 8-10 exercícios \\
\hline Intensidade & Cargas graduais. Começamos com autocargas. \\
\hline \multicolumn{2}{|c|}{ Trabalhar a flexibilidade diariamente. Estático e entre $10-30 \mathrm{~s}$} \\
\hline
\end{tabular}




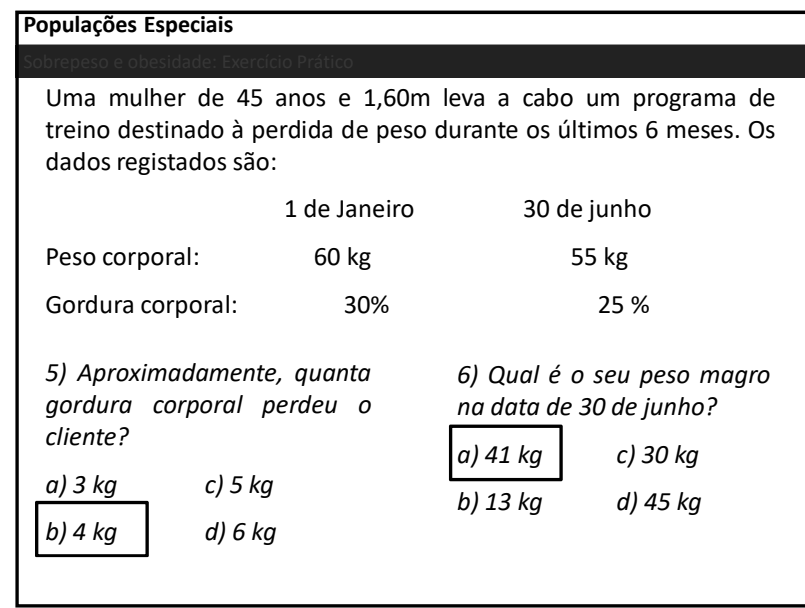

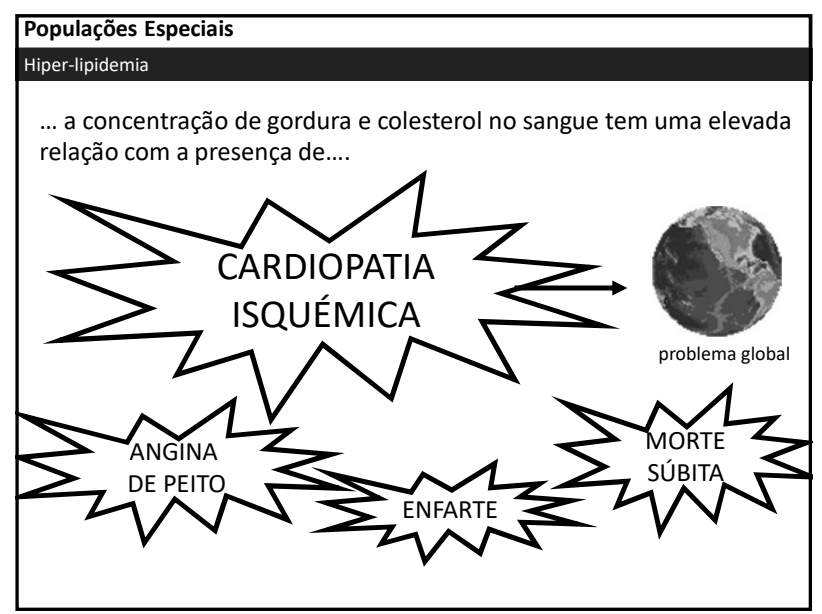

\begin{tabular}{|c|c|}
\hline \multicolumn{2}{|c|}{ Populações Especiais } \\
\hline \multicolumn{2}{|l|}{ Hiper-lipidemia } \\
\hline & $\begin{array}{l}\text { Clasificación ATP III de los niveles de LDL, HDL, } \\
\text { colesterol total y triglicéridos (mg/dL) }\end{array}$ \\
\hline & Lipoproteinas de baja densidad (LDL) \\
\hline$<100$ & Optimo \\
\hline $100-129$ & Casi optimo/por encima del nivel optimo \\
\hline 130-159 & Cercano a un nivel alto \\
\hline 160-189 & Alto \\
\hline$>190$ & Muy alto \\
\hline \multicolumn{2}{|c|}{ Lipoproteinas de alta densidad (HDL) } \\
\hline$<40$ & Bajo \\
\hline$>60$ & Alto \\
\hline \multicolumn{2}{|c|}{ Colesterol total } \\
\hline$<200$ & Deseable \\
\hline $200-239$ & Cercano a un nivel alto \\
\hline$>240$ & Alto \\
\hline \multicolumn{2}{|c|}{ Triglicéridos } \\
\hline$<150$ & Normal \\
\hline $150-199$ & Cercano a un nivel alto \\
\hline $200-499$ & Alto \\
\hline$>500$ & Muy alto \\
\hline
\end{tabular}




\begin{tabular}{|c|c|}
\hline \multicolumn{2}{|c|}{ Populações Especiais } \\
\hline \multicolumn{2}{|l|}{ Hiper-lipidemia } \\
\hline \multirow{3}{*}{\multicolumn{2}{|c|}{$\begin{array}{l}\text { Que fazemos com um cliente } \\
\text { com hiperlipidemia? }\end{array}$}} \\
\hline & \\
\hline & \\
\hline Frequência & 5-7 dias/semana \\
\hline Volume & 2 sessões/dia 20-30 min. Objetivo final: $40-60 \mathrm{~min} / \mathrm{dia}$ \\
\hline Tipo de exercício & Atividades que impliquem grandes grupos musculares \\
\hline Intensidade & $\begin{array}{l}40-70 \% \mathrm{VO}_{2} \max \\
\text { Monitorizar intensidade com PSE e trabalhar sobre 11-16 }\end{array}$ \\
\hline $\begin{array}{l}\text { Dufação } \\
\text { pißgrama }\end{array}$ & $\begin{array}{l}4 \text { meses para modificar a atitude } \\
9-12 \text { meses para alterações no perfil lipídico }\end{array}$ \\
\hline
\end{tabular}

\begin{tabular}{ll|}
\hline Populações Especiais & \\
\hline \multicolumn{1}{c|}{ Sesordens alimentares } \\
\hline
\end{tabular}

\begin{tabular}{|l|}
\hline Populações Especiais \\
\hline Sucede quando se tem 3 ou mais dos seguintes sintomas: \\
- Obesidade abdominal: circunferência da cintura $>102 \mathrm{~cm}(\mathrm{M})$ ou $88 \mathrm{~cm}(\mathrm{~F})$ \\
- Hiperlipidemia: $\geq 150 \mathrm{mg} / \mathrm{dL}$ triglicéridos \\
- Níveis reduzidos de $\mathrm{HDL}:<40 \mathrm{mg} / \mathrm{dL}$ (M) ou $<50 \mathrm{mg} / \mathrm{dL}$ (F) \\
- Tensão arterial elevada: $\geq 130 / 85 \mathrm{mmHg}$ \\
- Glicemia elevada em jejum: $\geq 110 \mathrm{mg} / \mathrm{dL}$ \\
Estas pessoas podem desenvolver níveis altos de insulina no sangue. \\
Desenvolve uma resistência à insulina (menor sensibilidade), pelo que as células \\
não respondem a esta hormona. Ao não introduzir a glicose nas células, a sua \\
concentração permanece elevada no sangue. \\
RISCO: de enfermidade coronária, de acidente cerebrovascular e de diabetes tipo \\
II.
\end{tabular}


Populações Especiais

Diabetes Mellitus

TIPOS:

- Tipo I (insulinodependente): afeta a 10\% dos diabéticos

- Tipo II (não insulinodependente): afeta $\mathbf{9 0 \%}$ dos diabéticos

\section{ALGUNS SINAIS e SINTOMAS:}

- Maior frequência de micção

- Aumento da sede

- Mais apetite

- Debilidade general
Populações Especiais Diabetes Mellitus

Contraindicaciones al ejercicio en clientes diabéticos

Glucemia $>240 \mathrm{mg} / \mathrm{dL}$ y cetonas en la orina en la diabetes tipo I

Glucemia $>300 \mathrm{mg} / \mathrm{dL}$ sin cetonas

Los clientes con retinopatía proliferativa deben evitar las actividades de intensidad

elevada y vigorosa

Nefropatia grave

Los clientes con pérdida de sensibilidad en los dedos (neuropatía periférica) deben

evitar caminar o trotar al aire libre: Se recomienda natación o ciclismo

Enfermedad, infección o fiebre agudas

Evidencia de una enfermedad cardiovascular subyacente que no se ha evaluado médicamente
Populações Especiais Diabetes Mellitus

\begin{tabular}{|l|}
\hline \multicolumn{1}{|c|}{$\begin{array}{r}\text { Signos y síntomas de } \\
\text { hipoglucemia }\end{array}$} \\
Sudoración \\
\hline Hambre \\
\hline Palpitaciones \\
\hline Cefalea \\
\hline Taquicardia \\
\hline Ansiedad \\
\hline Temblores \\
\hline Mareos \\
\hline Visión borrosa \\
\hline Confusión \\
\hline Convulsiones \\
\hline Sincope \\
\hline Coma \\
\hline
\end{tabular}

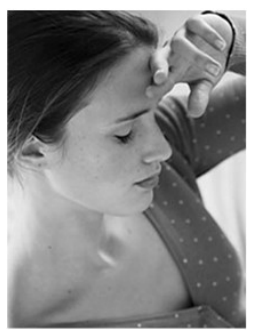




\section{Populações Especiais}

Diabetes Mellitus

ORIENTAÇÕES PARA O TREINO DA FORÇA

\begin{tabular}{|l|l|}
\hline Frequência & 2-3 dias/semana (não consecutivos) \\
\hline Intensidade & Cargas graduais. Iniciamos com auto-cargas. \\
\hline Volume & 8-12 reps/série \\
\hline № exercícios & $\begin{array}{l}\text { Pelo menos um exercício por grupo muscular (total 8-10 } \\
\text { exercícios) }\end{array}$ \\
\hline $\begin{array}{l}\text { Velocidade de } \\
\text { execução }\end{array}$ & Controlada \\
\hline
\end{tabular}

ORIENTAÇÕES PARA O TREINO AERÓBIO

\begin{tabular}{|l|l|}
\hline Frequência & $4-6$ dias/semana \\
\hline Intensidade & $50-85 \%$ da Fcmax. Se usamos PSE, 12-14. \\
\hline Volume & 20-60 minutos por sessão \\
\hline
\end{tabular}

Populações Especiais Diabetes Mellitus

\section{IMPORTANTE:}

Controlar os níveis de glucose antes e depois do exercício (e se possivel, durante)

- Não realizar exercício se os níveis são superiores a $250 \mathrm{mg} / \mathrm{dl}$ de sangue

Precaução com as hipoglicemias pre-exercício $(<100 \mathrm{mg} / \mathrm{dl})$

- O exercício deve ser programado 1-2 horas depois da comida.

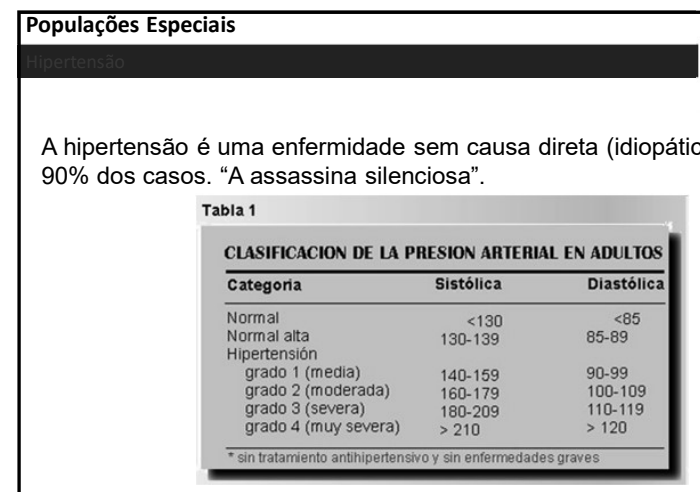

Tratamento da hipertensão: Fármacos e beta-bloqueantes

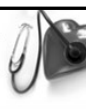

A hipertensão é uma enfermidade sem causa direta (idiopática) em 


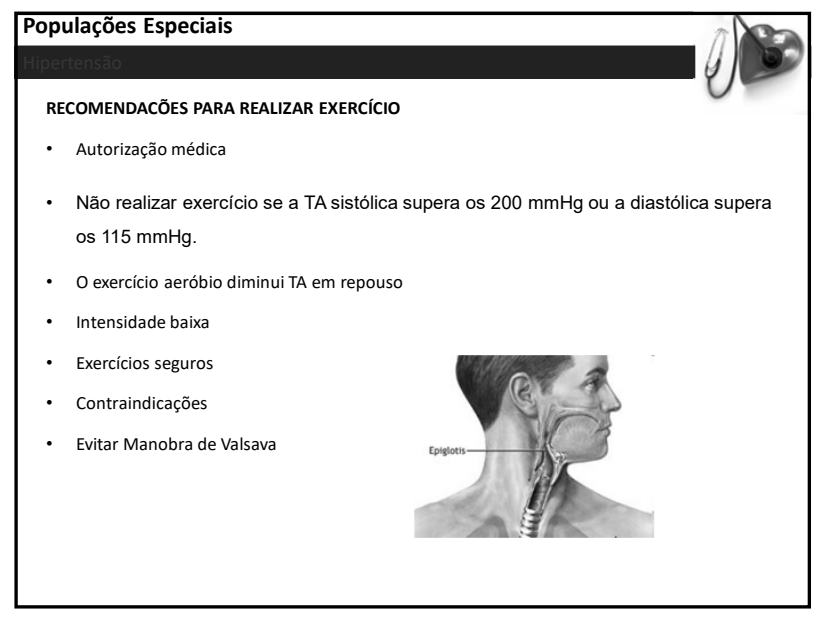

\begin{tabular}{l} 
Populações Especiais \\
\begin{tabular}{|l|l|}
\hline ORIENTAÇÕES PARA O TREINO AERÓBIO \\
\hline Frequência & $3-7$ dias/semana \\
\hline Intensidade & $\begin{array}{l}\text { Baixa/moderada (PSE de 8-10, limite 13) } \\
\text { de } 40 \text { até } 85 \% \text { Fcmax }\end{array}$ \\
\hline Volume & $30-60$ min \\
\hline Exercícios & Caminhar, bicicleta... \\
\hline Gasto semanal & $700-2000$ Kcal. \\
\hline ORIENTAÇÕES PARA O TREINO DA FORÇA \\
\hline Frequência & $2-3$ dias/semana \\
\hline Intensidade & $50-60 \%$ 1RM (primar pelo volume) \\
\hline Volume & $1-3$ séries e 16-20 repetições \\
\hline Descanso & $2-3$ ' \\
\hline Exercícios & Multiarticulares \\
\hline
\end{tabular} \\
\hline
\end{tabular}

\begin{tabular}{|l|}
\hline Populações Especiais \\
\hline Enfarte do miocárdio \\
- Músculo cardíaco morre potencialmente \\
- Autorização médica \\
- Objetivo: tarefas quotidianas \\
- No Manobra de Valsava \\
Objetivos a trabalhar \\
- Aumentar a capacidade aeróbia $\rightarrow$ capacidade para praticar \\
atividades de ócio e da vida diária \\
- Diminuir a tensão arterial \\
\end{tabular}




\begin{tabular}{|c|c|}
\hline Populações Esp & \\
\hline Enfarte do miocárc & \\
\hline ORIENTAÇÕE & TREINO AERÓBIO \\
\hline Frequência & 3 veces/semana \\
\hline Intensidade & Baixa/moderada \\
\hline & $40-70$ FCmax \\
\hline Volume & 5-60 min. \\
\hline Exercícios & bicicleta... \\
\hline ORIENTAÇÕE & TREINO DA FORÇA \\
\hline Frequência & 2-3 dias/semana \\
\hline Intensidade & $50-60 \% 1 \mathrm{RM}$ (primar pelo volume) \\
\hline Volume & 1-3 séries e 8-12 repetições \\
\hline Não avaliar & \\
\hline Trabalho de & ade, coordenação e equilíbrio \\
\hline
\end{tabular}

\begin{tabular}{l} 
Populações Especiais \\
1) As seguintes modificações ou objetivos dietéticos podem ser aplicados em todos os \\
clientes com excesso de peso, exceto: \\
a) Estabelecer uma meta de perda de peso de 10\% do peso corporal para os primeiros 6 \\
meses \\
b) Modificar a seleção de alimentos de forma a que reduzam a ingestão calórica e de \\
gorduras \\
c) propor-se a perder uma média de 0,5 a 1kg por semana \\
d) Seguir um plano de 1.000 calorias/dia \\
\hline
\end{tabular}

\section{Bibliografia a consultar}

Pescatello, L. S., \& American College of Sports Medicine. (2014). ACSM's guidelines for exercise testing and prescription. Philadelphia: Wolters Kluwer/Lippincott Williams \& Wilkin

Ferguson, B. (2014). ACSM's Guidelines for Exercise Testing and Prescription 9th Ed. 2014. The Journal of the Canadian Chiropractic Association, 58(3), 328.

LEE E. BROWN AND JOSEPH P. WEIR. ASEP Procedures Recommendation I: Accurate Assessment Of Muscular Strength And Power. JEPonline. 2001;4(3):1-21

Reynolds, J.M., T.J. Gordon, and R.A. Robergs. (2006). Prediction of 1 repetition maximum strength from multiple repetition maximum testing and anthropometry. J. Strength Cond. Res. 20(3):584-592. Garber, C. E., Blissmer, B., Deschenes, M. R., Franklin, B. A., Lamonte, M. J., Lee, I. M.,
Nieman, D. C., ... American College of Sports Medicine. (January 01, 2011). American College of Sports Medicine position stand. Quantity and quality of exercise for developing and maintaining cardiorespiratory, musculoskeletal, and neuromotor fitness in apparently healthy $1334-59$

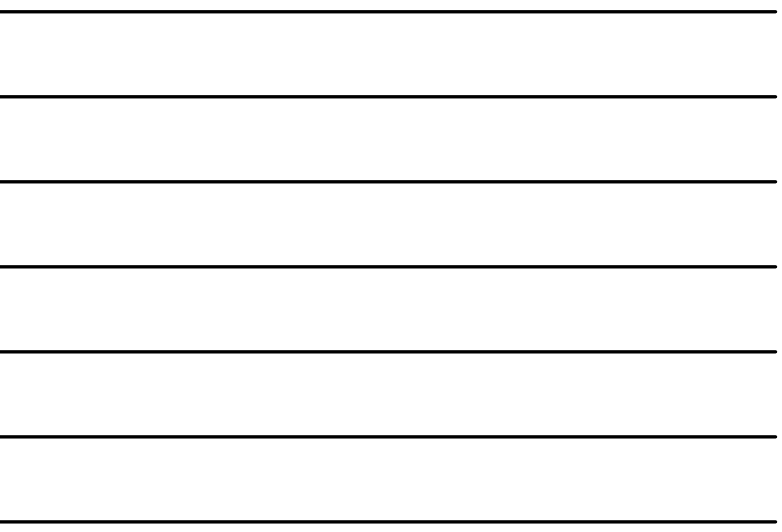

